\title{
Cave: Klostridien bei betagten Patienten
}

\author{
Am 8. Februar 2012 gab die amerikanische Arznei- \\ mittelbehörde FDA eine "Safety Information“ heraus, \\ die vor Nebenwirkungen von Protonenpumpen- \\ inhibitoren (PPI) warnt.
}

— Sechs in den USA gebräuchliche, teils frei verkäufliche, teils verschreibungspflichtige Prazole (Rabeprazol, Dexlansoprazol, Esomeprazol, Omeprazol, Lansoprazol und Pantoprazol), die als Nebenwirkung alle Clostridium-difficile-assoziierte Durchfälle (CDAD) verursachen können, werden aufgeführt.

Es wird geraten, dass Menschen, die PPI einnehmen und darunter hartnäckige Durchfälle bekommen, ggf. mit abdominellen Schmerzen und Fieber, sich unverzüglich in ärztliche Obhut begeben sollten. Auch vor einer Ausbreitung von CDAD, z. B. in Krankenhäusern, wird gewarnt.

\section{Kommentar}

Protonenpumpeninhibitoren (Prazole) zählen zu den am häufigsten verordneten Medikamenten überhaupt und werden, gemessen an ihrer fast ubiquitären Verbreitung, erstaunlich gut vertragen. In letzter Zeit mehren sich aber Berichte über unerwünschte Wirkungen. Eine Osteoporose begünstigende Wirkung mit vermehrtem Auftreten von Knochenbrüchen wird diskutiert. Weiterhin scheinen unter PPI vermehrt Infektionen aufzutreten. Sowohl ambulant als auch im Krankenhaus erworbene Pneumonien sind unter PPI-Einnahme häufiger. Ebenfalls durch PPI begünstig wird das Auftreten Clostridium-difficile-assoziierter Durchfallerkrankungen (CDAD), besonders bei älteren und gebrechlichen Patienten.

Vor dieser potenziell schweren Durchfallerkrankung wird nun offiziell von der FDA gewarnt. Andererseits haben PPI einen wichtigen und etablierten Stellenwert bei der Behandlung peptischer Erkrankungen. Um eine unnötige oder sogar gefährliche Gabe von PPI zu vermeiden, fragen wir bei unseren geriatrischen Patienten, die bei Krankenhausaufnahme häufig PPI in ihrer Medikamentenliste haben, nach einer Anamnese von Magen-/Duodenalulzera und oberen GI-Blutungen. Wenn solche in den letzten zwei Jahren nicht aufgetreten sind, der Patient keine NSAR einnimmt (besonders problematisch in Kombination mit ASS und/oder Kortikosteroiden und evtl. sogar noch oralen Antikoagulanzien) und keine Anamnese von Refluxkrankheit und Sodbrennen zu erfragen ist, stehen wir einem kontrollierten Weglassversuch des PPI aufgeschlossen gegenüber. Eine gute Kommunikation mit dem Hausarzt ist dabei aber erforderlich.

J. ZeEH =

- www.fda.gov/Safety/MedWatch

\section{Hier muss der Dummy durch eine Anzeige ersetzt werden !!}

